Reinventing Space Conference BIS-RS-2015-

\title{
Resource Prospector (RP): A Cost-Effective Lunar Resource Pathfinder
}

Daniel Andrews

NASA-Ames Research Center

Moffett Field, CA, 94035

USA 


\title{
Resource Prospector (RP): A Cost-Effective Lunar Resource Pathfinder
}

\author{
D. Andrews ${ }^{1}$ \\ NASA-Ames Research Center, Moffett Field, CA, 94035, USA
}

\begin{abstract}
I. Abstract
$\mathrm{T}$ he Resource Prospector (RP) is an in-situ resource utilization (ISRU) technology demonstration mission under study by the NASA Human Exploration and Operations Mission Directorate's (HEOMD). This clever mission is currently planned to launch in 2020 and will demonstrate extraction of oxygen, water and other volatiles, as well measure mineralogical content such as silicon and light metals, like aluminum and titanium, from lunar regolith. Efficient expansion of human presence beyond low-Earth orbit to asteroids and Mars will require the maximum possible use of local materials, so-called in-situ resources. The moon presents a unique destination to conduct robotic investigations that advance ISRU capabilities, as well as providing significant exploration and science value. This mission is equally important, however, for how it executes as a risk-tolerant, cost-effective mission. RP follows on the path-finding approaches of the Lunar Crater Observation and Sensing Satellite (LCROSS) mission. The LCROSS mission confirmed the presence of water-ice on the moon, but also established a new lightweight-approach to project and mission execution which was considerably cheaper and faster than traditional NASA missions.
\end{abstract}

RP has been designated as a "Class D" mission, just as LCROSS. This mission classification is the most risk-tolerant class of mission within the NASA risk framework and as such, is given more latitude to accept higher-levels of residual risk. The intention is that by saving monies normally spent attempting to assure a single mission's success, more missions can be funded. A well-designed portfolio can accept occasional mission failure, as its still gets more done for the same investment of resources. This classification enables tailoring the NASA Policy Requirements (NPRs) to "lighter-weight" approaches to mission management and execution.

$\mathrm{RP}$ is also assessing both international and commercial partnerships as a means to maximize return on the investment. International partnerships can provide both capabilities synergies and cost-sharing opportunities, while the evolving "new space" commercial options are revealing new approaches to acquiring cost-effective services, including

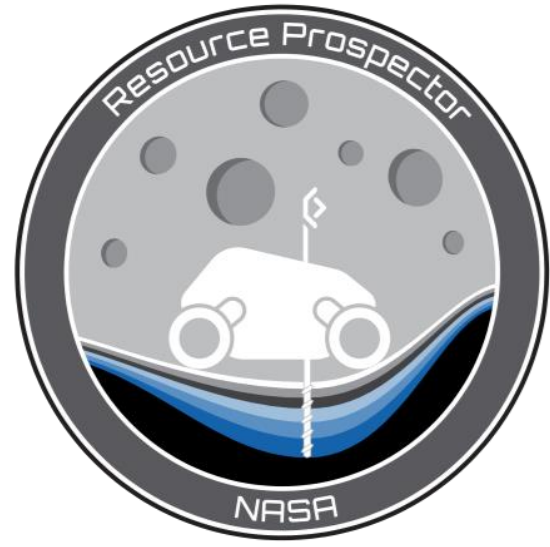

Figure 1. Resource Prospector team the benefits of "bundling" services. Even the world of launch vehicles is changing, offering much less expensive access to space, especially if NASA is able to be flexible in how it approaches mission assurance. Finally, leveraging investments being made elsewhere within a program portfolio, can enable cost-savings by enabling two applications with one investment.

RP will be the next pathfinder mission to both enable exploration capabilities for future missions, and continue to evolve cost-effective approaches for NASA.

\section{Reinvention}

The Resource Prospector (RP) is an in-situ resource utilization (ISRU) technology demonstration mission under study by the NASA Human Exploration and Operations Mission Directorate's (HEOMD). This clever mission is currently planned to launch in 2020 and will demonstrate extraction of oxygen, water and other volatiles, as well

${ }^{1}$ RP Project Manager, Programs \& Projects Directorate, Moffett Field, CA 94035 
measure mineralogical content such as silicon and light metals, like aluminum and titanium, from lunar regolith. Efficient expansion of human presence beyond low-Earth orbit to asteroids and Mars will require the maximum possible use of local materials, so-called in-situ resources. The moon presents a unique destination to conduct robotic investigations that advance ISRU capabilities, as well as providing significant exploration and science value.

This mission is equally important, however, for how it executes as a risk-tolerant, cost-effective mission. HEOMD was inspired by the impressive, path-finding approaches of the Lunar Crater Observation and Sensing Satellite (LCROSS) mission ${ }^{[1]}$. The LCROSS mission confirmed the presence of water-ice on the moon, but also established a new lightweight-approach to mission project execution which was considerably cheaper and faster than traditional NASA missions.

Resource Prospector has been designated as a "Class D" mission, just as LCROSS. This mission classification is the most risk-tolerant class of mission within the NASA risk framework and as such, is given more latitude to accept higher-levels of residual risk. The intention is that by saving monies normally spent attempting to assure a single mission's success, more missions can be funded. A well-designed portfolio can accept occasional mission failure, as its still gets more done for the same investment of resources. This classification enables tailoring the NASA Policy Requirements (NPRs) to "lighter-weight" approaches to mission management and execution.

This paper will attempt to frame some of the novel approaches taken by the Resource Prospector team as it continues Phase A development, inspired by the LCROSS mission to the moon.

\section{Resource Prospector Overview \& "RP15” Approach}

Resource Prospector is a Phase $\mathrm{A}^{[2]}$ project, managed within the Advanced Exploration Systems (AES) Division of NASA's Human Exploration and Operations Mission Directorate (HEOMD), currently planned for launch in 2020. The activities which apply to the RP flight plans and hardware are collectively referred-to as "RP" - the mission which will fly. However, in the 12-month span of fiscal year 2015, the RP team has stepped-up of also building a terrestrial Engineering Test Unit (ETU) "surface segment" to be used to mature technologies, perform risk reduction, and practice how we would operate the actual mission. This ETU rover/payload surface segment will be taken from conceptual Needs, Goals, and Objectives (NGOs), which were agreed-to with NASA-HQ, to creating a working rover/payload ETU in a single year! The great challenge of building this ETU will promote learning and enable risk reduction activities to take place, and is referred-to as "RP15".

Both RP15 and RP seek the same functionality in general; however, RP15 is limited by both programmatic and terrestrial constraints. RP (the flight mission) is designed to prospect the lunar surface, create a map revealing the nature and distribution of the volatiles, and perform an early demonstration of materials processing while on the lunar surface. RP15 enables early testing of some of the most important capabilities required to execute the flight plan of RP. RP mission functionality and RP15 test functionality is illustrated below.

1. Mapping the Surface. The RP15 terrestrial rover will provide mobility enabling roving over surfaces and slopes analogous to what will be expected on the polar regions of the moon. The force of gravity is different for our RP15 terrestrial ETU, but is still relatable to what will be experienced on the moon. Further, the rover system is being designed to enable testing in gravity off-load facilities to see how it performs in a true $1 / 6 \mathrm{~g}$ environment. This roving platform will carry both the Neutron Spectrometer System (NSS) and Near Infrared Volatiles Spectrometer System (NIRVSS). During RP15 field testing the NSS is included to replicate packaging constraints, but will not be

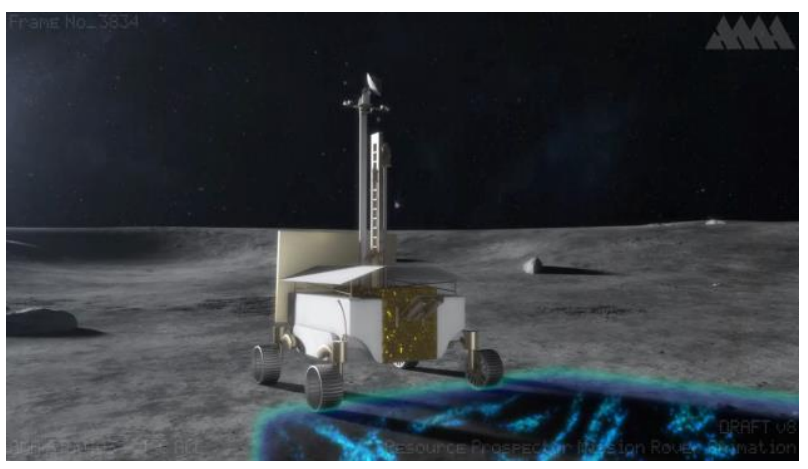

Figure 2. RP scanning for subsurface volatiles functional; however, the NIRVSS instrument will be functional, enabling sensing/measuring the indigenous soil and practicing the prospecting part of the mission. Figure 3 illustrates how this scanning would work during actual lunar roving. 
2. Entering Permanent Shadows: We envision RP15 will navigate terrestrial "Permanently-Shadowed Regions" (PSR) by either testing at night in the rock yard, or by testing in a high-bay with darkened conditions. The degree of fidelity (regolith simulant, volatile doping, etc.) is negotiable based on resources available, but this testing could aid in understanding navigation, positioning, and measurement difficulties in rover-only lighted conditions. Figure 4 illustrates the RP rover charging in the Sun prior to entering a PSR on the moon.

3. Exposing Regolith: The Drill system is also included on the RP15 ETU, enabling actual drilling operations from

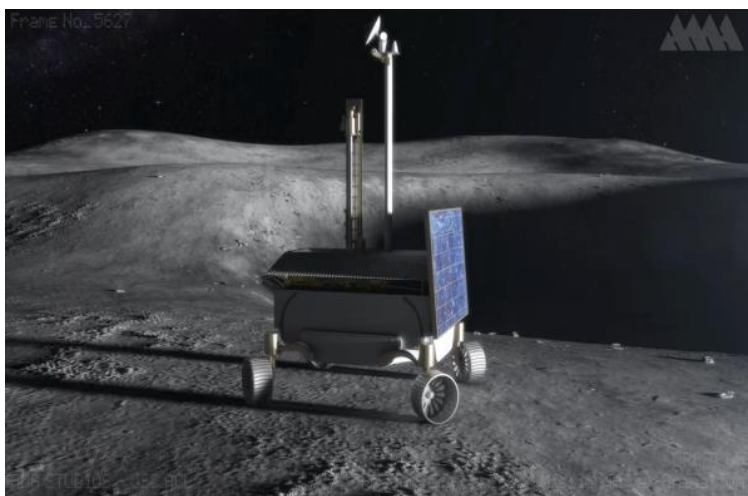

Figure 3. RP prior to entering a PSR the rover. This will enable testing procedures and methods for drilling and capturing tailings from the drill bit, even while acknowledging this testing will be in a $1 \mathrm{~g}$ environment. We expect to learn about constraints on the rover system while drilling on slopes, which side-loads the drill bit, potentially causing binding. The rover suspension system will be required to compensate for loads which move during these drilling conditions, enabling extrapolation of what might be found in the $1 / 6 \mathrm{~g}$ environment of the moon. Further, the NIRVSS instrument will measure the volatiles excavated from the soil to the surface. We envision having specifically-prepared sample tubes, perhaps buried in the lunar rock yard, wherein the rover can drive over the sample tube, drill/excavate and measure.

4. Capturing and Heating Regolith: The same drill which provides the excavation capability illustrated above also has the capability to acquire samples from as deep as $1 \mathrm{~m}$, into a sample handling system. The samples are then deposited into the Oxygen and Volatile Extraction Node (OVEN) Subsystem, evolving the volatiles by heating the regolith in a sealed chamber and extracting oxygen and hydrogen from the regolith sample. RP15

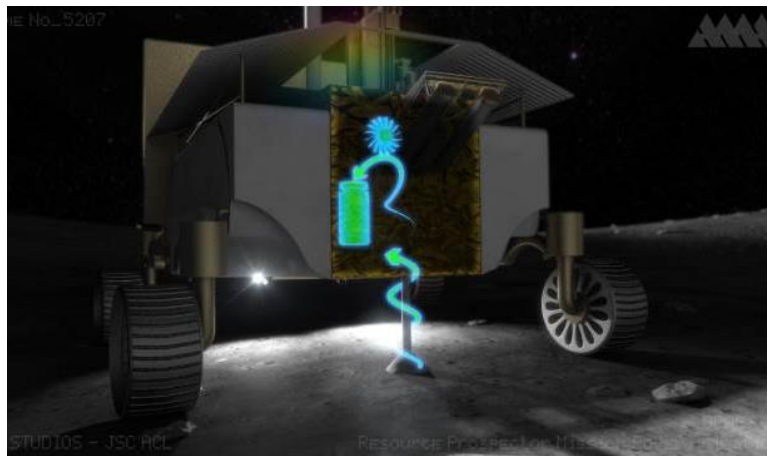

Figure 4. RP drilling for subsurface volatiles

will be carrying an ETU version of OVEN, capable of performing similar functions to the flight version of the same subsystem. This includes the ability to heat the regolith samples to $150-450 \mathrm{degC}$ to liberate the volatiles for measurement. Figure 5 illustrates the RP rover performing a drill operation and bringing material from $1 \mathrm{~m}$ below the surface up into the OVEN system.

5. Identifying the Volatiles: The RP Lunar Advanced Volatile Analysis (LAVA) Subsystem is also included in on RP15. This system will analyze the effluent gas/vapor from the OVEN Subsystem using gas chromatograph and/or mass spectrometer sensor technologies. Volatiles evolution from material in the doped sample tubes will enable verification of the design approaches planned for RP.

\section{Reinventing the Approach}

The previous section describing the RP mission illustrates the complexity of this mission, and yet the team has been challenged to approach mission development differently; more cost-effectively, similar to what was accomplished on the LCROSS mission. To do so requires reinventing the approach to how missions are traditionally done, and even requires the customers to understand some of the ramifications of different approaches.

Spaceflight is historically expensive. The best starting point for making a mission more cost effective and streamlined is to challenge the notion of what is required to accomplish the mission. Space missions suffer from three circular maladies: They traditionally do not deal in volume production so they are expensive; because they are 
expensive, they "cannot fail"; since they cannot fail, their success must be assured... which is expensive! The result is mission designers spend a lot of resources attempting to assure a mission succeeds because they are too expensive to fail. However, there are ways to help strike a more efficient balance, an area in which LCROSS was a pathfinder.

Performance: The first step is to eliminate the pursuit of maximum performance. Maximum performance requires non-traditional, sometimes heroic activities which do not come cheap. Further, maximum performance can carry exotic parts requirements which do not usually come cheap. If the mission doesn't require being a technical marvel, be mindful of your design's sophistication. LCROSS was not a Faster, Better, Cheaper mission; it was a Faster, "Good-enough", (and therefore) Cheaper mission. LCROSS strived to be a high-heritage, low-complexity, and just good-enough mission. The spacecraft was simple by design - an intentionally low-risk approach.

Risk-Tolerance: Further enabling cost savings is the risk classification of the mission. NASA carries four risk classification levels: A, B, C \& D, wherein Class A is the least risk-tolerant (expensive flagship or human spaceflight missions), and Class D is the most risk tolerant (smaller robotic missions). LCROSS and RP are both Class D missions. Within NASA this risk classification enables taking-on taller technical challenges, or, performing to tighter programmatic constraints like cost and schedule. This risk-tolerant classification enables "single-string" designs, which means that there is no required redundancy in the design. It's acceptable for the system to fail if a single event within the system occurs. This approach allows the mission designer to save mass, cost, and schedule by streamlining both the design process and the complexity of the system, no longer requiring redundant systems.

There is an important tie, however, to the Performance topic above. Simply accepting additional risk is not necessarily desirable unless really trapped in a schedule or cost box. More ideal would be to directly attack mission and design complexity, which is another way of stating that you should not eye making a technical marvel, but reliable, simple designs. These are far more likely to work the first time and continue to work than sophisticated designs. Since the design is likely also single string, having fewer ways the design can fail you is a nice compensation for not having expensive backups systems. Put another way, as a system becomes more complex, it is more susceptible to human design and test errors, and less likely to achieve success. The key is to keep the mission scope as small as can be tolerated.

Design to Cost: This is another way to look at the Performance topic. Instead of looking to "what is technically possible" with a mission design, look to what capabilities exist to minimally achieve the mission goals. Instead of pushing the limits of technology or performance, do as much as you can within the capabilities of a minimal system. This is how LCROSS operated in the conceptual phase of project development. The team understood that custom development is fraught with risk and can be costly by taking a lot of time in the design and testing phases. LCROSS was a design-to-cost ${ }^{[3]}$ (DTC) project, working to cost and schedule constraints, the principal drivers for the project. By dealing as much as possible with existing designs, we had a set of capabilities with which to work, and that helped to contain cost and schedule.

RP will be taking the approaches found by LCROSS and applying them where appropriate, but RP is an example of a mission where the stated goals are fairly challenging, and more sophistication will have to remain in the system. Our challenge will be in deciding where we can be leverage what exists and what simply doesn't exist and requires custom development. However, even in those latter cases, we will always be eyeing simplicity everywhere we can find it.

Risk Retirement: In those cases where custom designs are required, retire risk as soon as possible. Risks that are left to fester later and later into the system design get more and more expensive to root-out later. RP is very much taking this approach with "RP15", discussed earlier. RP15 is an ETU to learn about the design intentions in Phase-A when it is much easier and less costly to change approaches in the interest of cost savings. The RP team has effectively gone through an entire design and test cycle with RP15, yielding many, many useful results for both simplifications, and design intentions which didn't work the way we thought. RP15 enabled us to retire both schedule and technical risk early, and should enable us to streamline moving through the typical mission development phases, increasing speed and reducing cost.

Risk retirement also means evaluating the degree to which testing is required - or at all. If you are working in a costconstrained environment, you probably cannot afford to do more than what is essential to meet your overall goals. 
With a keen understanding of the nature of your risks, you should be able to estimate the likelihood and consequence of the risk occurring and attempt to have rough parity between your other mission risks. True, there may be some risks which you would like to treat with some special attention, but in general, the overall posture of your risks to be pretty self-consistent, as there is no value in having one system have a $98 \%$ confidence of working, when another system, maybe your communications system, has only a $70 \%$ confidence... If your communications system fails on the mission, it doesn't likely matter that the other systems are working beautifully, as you are no longer able to communicate with the spacecraft! This risk parity approach could enable saving some time and money by actually choosing to do less.

Here's an excellent example from the LCROSS mission involving environmental testing. The LCROSS spacecraft, like any spacecraft needed to go through environmental testing to provide some confidence that when the mission is launched and activated, it will power-up and move-out. The problem is that environmental testing can be very expensive, as they sometimes involve very large, expensive facilities, large power sources and some exotic gasses and temperature which can make the testing quite expensive. Thermal-Vacuum (TVAC) chamber testing is probably about the most expensive because this type of testing requires a large facility (large enough to house your spacecraft), which can drop to temperatures and pressures the mission is likely going to see in space, but also during launch. There might even be additional tests combined with the TVAC test which bring additional complexity and therefore cost. For example, if you wished to simulate lighting conditions as well wherein you need a high-powered, solar-spectra light panel shining on one side of the spacecraft to testing power systems as well. The possibilities are endless, but those possibilities certainly drive cost as well. So how much testing is enough? "Traditional missions can run as many as 7 or 8 full thermal cycles, or more, to attempt to make sure the systems will behave as planned, taking potentially weeks or months to conduct this testing (depending on the size of the facility), and racking-up quite a bill. LCROSS studied white papers looking for guidance on TVAC thermal cycles and discovered that while many cycles can get you 95\% confidence on your system, most all of the workmanship problems on the spacecraft are revealed in the first full cycle of testing ${ }^{[4]}$ ! Because the LCROSS design was intentionally making high-use of proven parts, tested at lower levels, or already proven with the residual risk accepted, the largest risk we carried going into TVAC testing was that something was not built or assembled correctly. Inspections can help alleviate that risk, but if you really want to know if it's ready, TVAC testing is the way to go. Given the white paper conclusion about workmanship issue detection, LCROSS proceeded to plan for a single cycle of TVAC testing, later to evolve into 1.5 cycles. Here's what we did: He closed the chamber at room temperature and pressure and then pumpeddown to vacuum, and proceeded to heat the spacecraft. The idea was that any contaminants or volatiles which might still be on the spacecraft would be "cooked-off" during the hot cycle. We soaked there getting the spacecraft to a steady-state condition, and then proceeded to cool the chamber-down to levels we'll see in space, at a pace which matched the launch assent profile so that we could actually have the spacecraft dry-run what it will see during launch - a clever, additional verification. We then "cold-soaked" at that temperature since this will be the environment in which the spacecraft will spend most of its mission time. The spacecraft was then returned to ambient conditions, allowed to stabilize and it was removed from the TVAC chamber - all in less than a week. It's estimated that this short test could have saved several-hundred-thousand \$USD.

Resource Prospector is a different kind of mission than LCROSS in that it is having to work with more custom designs, given the complicated nature of the mission. The RP team will have to look carefully at trading between subsystem level testing and whole-spacecraft level testing and determine the nature of the residual risk going into TVAC testing. This evaluation will define how much time and how many cycles are needed to relieve RP risk looking toward flight.

Stakeholder Reporting: All of the ideas presented thus far have been technical topics, but cost drivers are not always technical in nature. If the stakeholder environment carries reporting-burdens which are heavy with detail and frequent in delivery it might be surprising to some just how much effort is being spent pulling all of that together. Further, you are taking the team off of their real purpose which is to design the system - or worse, you hire many more people to handle this activity in an attempt to minimize bothering the designers - driving cost! It's a vicious cycle which is sometime difficult to quantify, but when you witness a truly lightweight reporting environment, you can feel the difference.

LCROSS and RP both have customers who felt that typical NASA reporting and oversight caused more problems than they solved for Project teams and their execution. Lightweight reporting for LCROSS and RP includes a single, monthly report with simple "Quad charts" to capture the data. When you compare this to missions in which I've 
witnessed monthly 3-day MMRs (Monthly Management Reviews), wherein the whole team essentially stopped to participate, not to mention the work building the charts in advance... The reporting became a significant "product" of the team. Every stakeholder community will be different and every PM will have different levels of depth required to manage a project, but our LCROSS approach flows from the ideal that every piece of work you do should be helping move the project forward or you are wasting resources. That ideal is difficult to fully achieve, every activity spent on non-valuable work is displacing some other activity, and it is important that reporting not take on a life of its own. Frequently, reporting becomes something which must be $f e d$ and the reporting is not allowed to focus on what is needed to enable the project.

\section{Reinventing the Source}

There are traditional sources for space missions and then there are alternative source which might enable a flight project to be more efficient. The use of the term, "source" is loosely used here since it can apply to hardware, instruments or even people. This section will illustrate some of the opportunities employed in the past and under consideration for RP.

Unexpected Sources: LCROSS was a pathfinder for cost-constrained missions, certainly making use of its Class D designation to stay within the cost box, but also recognizing that we wanted the flight project to be successful. Part of our answer was to look to Commercial Off The Shelf (COTS) instruments and some flight-proven instruments, such as LCROSS' visible camera. We looked to well-established instruments from the commercial and industrial world to see if we could use them on the LCROSS mission. The instruments would ideally be ruggedized to improve their chances of survival in the LCROSS launch and space environments, confirming that fact by testing them in relevant environments (vacuum, temp extremes, vibe, etc.). This relationship with COTS vendors was interesting and synergistic, as the vendors were very interested in seeing their instruments get tested by NASA, and were quite accommodating in providing support when we found issues. It was a classic win-win. In the end, most instruments did very well in flight environments. There were some small issues which were easily handled such as one instrument test failed because a small bolt in an electronics box came loose during vibe testing. No adhesive had been applied to the bolt threads to help secure it for a dynamic loads environment. Once the adhesive was applied, the device passed testing just fine. In another case, an internal cable came loose in the instrument, because it was not staked down. We reduced the length of unsupported cable by staking it, thereby decreasing the cable strain experienced during the launch environment.

The selected suite of LCROSS instruments included a thermal camera (MID-IR1), which has been used in motorsports applications; Near-IR spectrometers (NSP1 \& NSP2) which are used in beer-making and carpet fiber analysis for assessing recyclability; UV visible spectrometers (UVS) from standard bench-top laboratory equipment; a visible camera routinely used in shuttle launch imagery; and Near-IR cameras (NIR-cam) used in fiber optic communications applications.

We applied this approach elsewhere on LCROSS, making use of surplus flight hardware to save money. For example, we discovered an existing, surplus TDRSS satellite propulsion tank, which with some simple modifications, became the main propellant tank on LCROSS! We even used a TRIANA satellite Inertial Measurement Unit (IMU) in the LCROSS attitude control system design.

In the end, this suite of instruments and flight hardware was cleverly applied on the LCROSS mission, which in the end, worked flawlessly, while saving a lot of money and time. RP is now taking a similar approach, where feasible, to decreasing cost and technical risk by flying a modified version of an LCROSS spectrometer, which has now also been utilized on the LADEE mission. The body of knowledge on this instrument is quite high and so the risk of deploying on RP is small. We're also taking battery technologies, cells and designs from the Robonaut robot and scaling the design to work for RP. These batteries have already been approved for deployment on the International Space Station (ISS), in and around humans, which saves a considerable amount of labor attempting to qualify a new battery design. Additionally, there are numerous rover steering system design which are inspired by the Chariot human rover, and the basis of the flight software (FSW) comes from the same software built and successfully flown on the LADEE mission. Each of the leverages come with cost savings because the development already exists.

Commercial Competitiveness: Related to the use of COTS hardware, is the benefit the marketplace has on driving 
costs down and reliability up. NASA is famous for designing sophisticated, capable instruments for its missions, but pays dearly for them in doing initial developments, investing all the non-recurring costs to bring a concept to reality... but what if there is an instrument out there which accomplishes $80 \%$ of what was needed on the mission? Sometimes that last $20 \%$ is essential for the mission, but I bet many times it is not and a satisfactory and wildly cheaper instrument deployment could be had. Commercial entities are most effective at drilling-in efficiencies once a technology is demonstrated, which ultimately translates into cost savings for all subsequent users - even if not originally designed for your application. The previous discussion from the LCROSS mission instruments is a great example of how to implement this approach.

Another frequent topic of both cost and risk is the use of Electrical, Electronic, and Electromechanical (EEE) parts in your spacecraft designs. Fifty years ago when electronic parts were starting to become mainstream, the quality of those part's manufacture was found to be highly-variable and at times "garbage". They certainly were not something to be relied-upon to run important systems, so as military spaceflight hardware. This reality gave birth to the advent of "Military grade" parts manufacture, along with requisite design hardening, test-to-failure parts-screening, and lot testing to make sure these parts could be relied-upon for something as important as national defense or something as expensive as a spacecraft. All these quality and reliability improvements, however, drove costs skyward making military and spaceflight hardware necessarily expensive, as you just couldn't take a chance...

An interesting thing then happened, however, over the next couple decades... the commercial world increasingly used electronics in their products, increasingly their sophistication, but also increasing the demand for good quality electronic componentry. One bad choice of a vendor could mean the end of a product line, or even a whole company, if the products failed. This created a natural pressure on the EEE supply chain to increase the quality control and overall reliability of even "commercial" parts - not because the free world depended on it, but because the company's reputation did! Can you imagine if it was routine for a cell phone manufacturer today to have $10 \%$ of their phones not work out of the box, or break if they were slightly mishandled? That company would vanish from the commercial marketplace in no time at all... and that possibility has driven-in unbelievable quality to commercial grade parts. However, many spaceflight missions still automatically require military-specifications (MilSpec) parts as a default. Now don't misunderstand this point: I am not saying all MilSpec parts can be replaced by commercial grade parts. I'm simply illustrating that there are lower-cost, higher-availability options which should be considered. Space missions which will endure long times in space with long exposures to radiation are likely going to still require radiation-hardened "Rad Hard" parts; however, if you have a limited-life missions, or can protect your electronics through other means, commercial grade parts are something to take a look at. LCROSS used commercial-grade components in most of its instruments, because they were simply commercial instruments, intended for use in a laboratory or in automotive applications - not space. However, LCROSS subsystem environmental testing was able to show these instruments were tough-enough, and if encountered a radiation fault, would simply reboot and then resume measurements. If the whole instrument were compromised, the overall instrument suite was robust-enough to tolerate the loss.... Which is exactly how we made this commercial approach work to our cost advantage. This risk-tolerant approach enabled us to rewrite the story of the "waterless" moon!

Buying in Bulk: This reality is probably self-evident, but there is considerable savings when acquiring goods in bulk since the supplier is able to consolidate activities. In my experience, NASA hasn't really attempted to take advantage of this reality. This has recently become apparent in my detailed surveying of NewSpace commercial entities and their ability to profitably-support lunar activities: launchers, LEO-delivery, Translunar LLO-delivery and lunar landing. While at the time of this writing lunar landing has not yet been accomplished by a private entity, many companies are aspiring to do just that, and as you might expect, are offering capabilities that are significantly discounted if you buy a large percent of the payload capacity, with maximized savings if you buy all of the capacity!

This pricing reality introduces some interesting twists if the buyer is willing to be a broker of the bulk payload buy. I encountered scenarios from some companies where I could nearly pay-off my costs for the part of the payload I was originally seeking by selling-off the excess payload at small-payload rates. The customers who bought those smaller payload opportunities would be satisfied with the results, and I would recover enough funding to nearly pay for my payload - for taking-on the role of broker. I actually briefed this concept to senior leaders within NASA, and while they understood the economies of scale and brokering, there was little interest to committing to this role, even with the given savings. I am sure there are varying reasons for not being interested in this additional brokering role, but remain confident that for those who would be willing to take on this role, there are very real cost-savings opportunities. 
Buying in Bundles: A related topic to Buying in Bulk, is Buying in Bundles. In this case, there isn't a bulk quantity being offered, but there is a bundle of services being provided. In the case of RP, I was doing market research on the readiness of the commercial marketplace to provide lunar landing services. This is different than the traditional model, where NASA buys a launch vehicle and pays a company to affix it atop the launch vehicle and then NASA works with the vendor to launch it and then separate the spacecraft and the NASA mission proceeds to its destination under NASA control. No, this model is much closer to a trucking service, commercially acquired. In this case, NASA acquires a lunar delivery service wherein NASA provides the specifications of what it wants to have delivered to the surface of the Moon, and then a commercial entity bids on that service, and NASA simply shows-up with its payload, and the commercial entity takes it, says thank you, and then promises to deliver it to location $(\mathrm{X}, \mathrm{Y})$ on the surface of the moon.

What is interesting about this possibility is that a similar, but different dynamic is occurring in the commercial process. In this case, the commercial vendor has to figure-out all the coordination of services so that it is and end-toend capability to NASA, but that also means that the commercial company can works deals, bulk-buys, and consolidate savings and pass them onto NASA is ways NASA could have never realized. One stark example has to do with the cost of launching spacecraft. NASA establishes provisions, riders, requirements on launches of its spacecraft which drive commercial pricing quite-high - as much as $40 \%$ higher in some cases. However, if NASA were to enable the commercial bundler to work deals with launch providers without the NASA additional requirements, there are considerable savings which can be passed-along to NASA as an end-buyer.

I've started to see hints of even deeper bundling coming wherein NASA wouldn't simply buy lunar landed mass services, but could buy lunar rover hosting services, where NASA simply places its instrument on a commercial rover and the commercial entity does everything from launching, to landing, to deployment, to roving and NASA simply takes data from their instrument and the commercial partner provides "the pipe" to send the data back to Earth for NASA use. Heck, I can see buying "data services" wherein NASA provides nothing but data requirements and the whole mission is bundled. Imagine the cost savings.

\section{Rediscovering Partnering}

Partnering is a term which is currently enjoying political favor. It sounds good to be partnering with others to achieve shared/blended objectives which can serve many different political needs; however, my interests are more with the direct benefits to the spaceflight project as a means to reinvent how to think of shared benefit. One obvious benefit of partnering is the "potluck". In a potluck dinner, all the invitees who come for dinner bring a dish to share themselves, and once everyone arrives a wide-variety of food is available and yet the only financial investment by each attendee is the cost/labor associated with the dish they brought. This analogy holds for partnering on a mission.

Launch Partnering: It's well understood that launch vehicles are expensive, especially when the mission is having to carry the entire financial burden of the launch. This is another place where partnering can help. LCROSS and LRO shared an Atlas V launch vehicle, for example, and it enabled the Exploration Systems Mission Directorate (ESMD) to get two missions from the cost of one launch vehicle. In the LRO/LCROSS scenario, both projects were funded by the same customer, but that isn't always the case. Two or more customers can split their payloads on a single launch and benefit with economies of scale of a larger launch vehicle. In the extreme case, very small spacecraft such as cubesats can either fit in the margins (small available space) of larger launches, or broker out an entire launch with dozens to hundreds of cubesats manifested on a single launch.

Pooled Capabilities Partnering: Pooling similar requirements into a single requirement set can bring savings to both missions. Two lunar missions, for example, both need a launch vehicle and a lander to begin their missions on the surface of the moon. The launch vehicles and landers will need propulsion systems, communications systems, power systems, structural systems, etc., and all of that will need to be tested before deployment. They will need a launch window, a launch site, frequency allocations, mission designers, etc., in order to successfully get to the surface of the moon. All of those facets (and many more) need to be paid-for. Now if those missions can be comanifested on the same launch and come to agreement on the nature and requirements of their trans-lunar journey, many of those required capabilities will be bought once, but enjoyed by two separate missions. Yes, two missions carry more mass and you'll pay for more propellant, and the structural design will have to support two missions instead of one, but 
all-in-all, there is very real savings enjoyed by the two missions in combining their requirements into a single requirements set for the launch and lunar delivery services provider.

Mission Element Partnering: RP's plan is to partner on its lunar lander needs, with NASA providing the rover and ISRU payload, as well as the launch vehicle, and has been in discussions with both international and commercial entities to see if there is shared value in doing so. The RP lander partnership concept was originally driven by purely political needs, but it carries with it very real cost benefits from the potluck metaphor. The intention would be to find a partner wherein building a lunar lander is something they would like to do to satisfy their own exploration goals. This lander would carry the RP surface segment rover, which would then prospect the surface of the moon. NASA brings very real money investment to that mission and the lander partner would as well, and both parties share other's data/results, making the investment doubly worthwhile. For example, one partner may need instrument A to take measurement A, but the other partner might be able to make use of Instrument A to take measurements B and C (i.e. a completely different use of Instrument A). It is not only cost-efficient, but also saves mass, volume and a number of technical measures. This is a current topic under discussion by RP and its partners regarding spectrometer measurements.

\section{Conclusion}

RP will be the first ISRU demonstration on another planetary body, taking first steps to be able to "live off the land". RP is in Phase A Formulation, having successfully passed its NASA Mission Concept Review (MCR) in the Fall of 2013. It is actively involved in international partnership discussions, to maximize return on this novel mission, within budgetary constraints. NASA will be providing the ISRU payload instrumentation, including drill, the roving mobility platform and launch vehicle. The lander will be provided either through an international partnership, or through a commercial relationship for an earlier, smaller demonstration prior to a full RP mission. The mission is scheduled for a 2020/21 launch, and NASA is working to a cost-effective $\$ 250 \mathrm{M}$ budget (not including the launch vehicle).

Most recently, the Resource Prospector

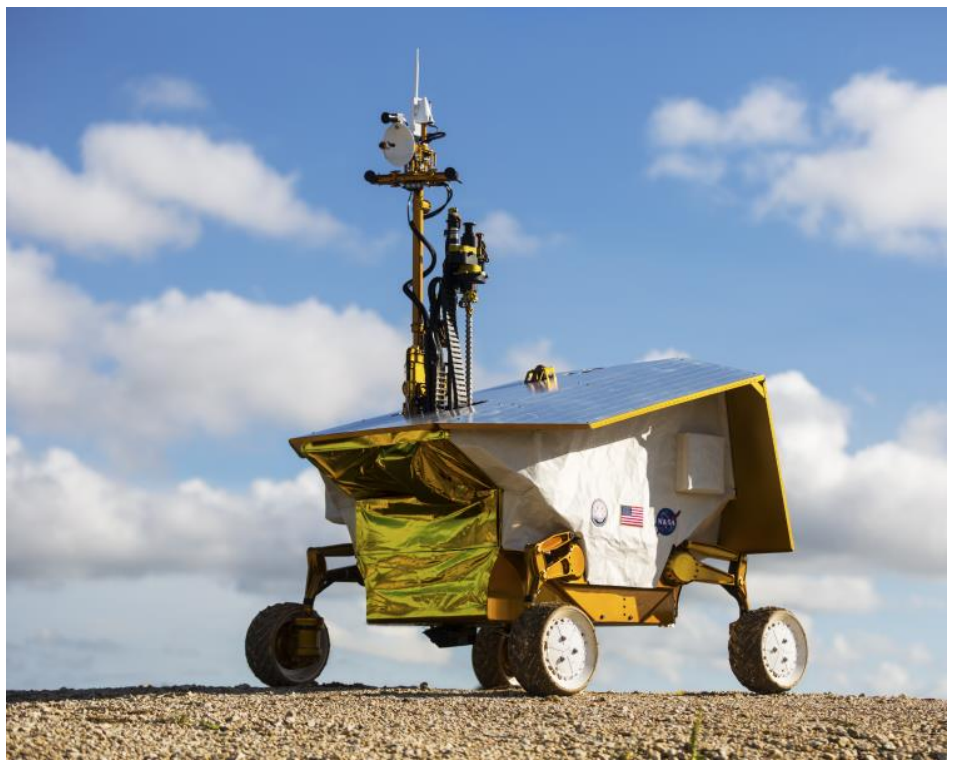

Figure 5. RP15 Terrestrial prototype, designed and built in a single year

team completed the design and build of "RP15"[5], a mission-in-a-year build of an entire terrestrial rover/payload system. RP15 is a Phase A "deep dive" ETU development, moving from concept to working hardware roving in a lunar-analogue rock yard in a single year. Through subsequent testing in 2016, RP15 will help reduce risk and improve resource prospector designs and approaches.

The NASA stakeholders have classified this mission as "Class D", which is the most risk-tolerant mission classification currently at NASA; this was the same mission classification as LCROSS ${ }^{[8]}$, which successfully rewrote the history books regarding water and the moon. RP looks to extend those cost-efficient, risk-tolerant approaches to a larger mission, while answering fundamental questions to enable future human exploration.

The great work of this RP team can be followed on the web at: www.nasa.gov/resource-prospector and on Twitter@NASAexplores.

\section{Acknowledgments}

The authors would like to thank the hard-working and immensely capable Resource Prospector team, across the many participating NASA Centers. Simultaneously working a Phase A mission towards flight, while also taking a 
terrestrial ETU rover/payload system from concept, design, build, test, and operations is an impressive feat. This work greatly reduces mission-level risk and matures concepts well beyond Phase A maturity. Additionally, we would like to express gratitude to our customers within the Advanced Exploration Systems Division of NASAHEOMD as well as the Game Changing Division of NASA-STMD, who asked this team to step-up to this important challenge, while encouraging lightweight approaches to mission management.

\section{References}

${ }^{1}$ Andrews, D. R., "LCROSS - Lunar Impactor: Pioneering Risk-Tolerant Exploration in a Search For Water on the Moon," $7^{\text {th }}$ International Planetary Probe Workshop (IPPW-7), Barcelona, Paper IAC-11-A5.1.4, 2011.

2 Andrews, D., Colaprete, A., Quinn, J., Chavers, D., Picard, M., "Introducing the Resource Prospector (RP) Mission", American Institute of Aeronautics and Astronautics (AIAA) SPACE 2014, San Diego, CA, USA.

${ }^{3}$ Atkins, K., "How to Plan and Manage Reserves Effectively," IEEE Aerospace Conference Proceedings, 2004.

${ }^{4}$ Quintero, A., Welch, J., "Perceptiveness of Thermal Vacuum Testing" $18^{\text {th }}$ Aerospace Testing Seminar, March 16-18, 1999.

${ }^{5}$ Andrews, D., Colaprete, A., Quinn, J., Bluethmann, B., Trimble, J., "Resource Prospector (RP) - Early Prototyping and Development", American Institute of Aeronautics and Astronautics (AIAA) SPACE 2015, Pasadena, CA, USA, Aug 2015. 